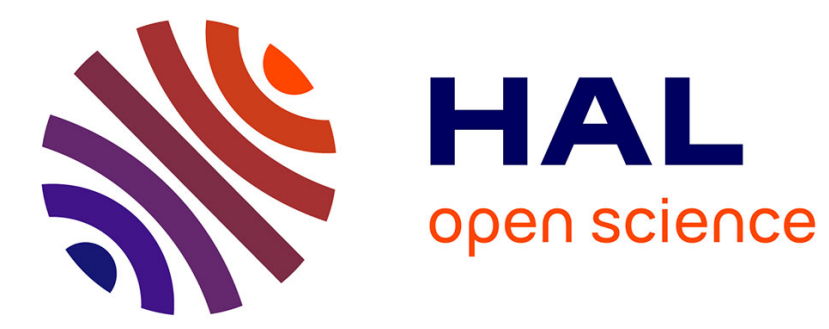

\title{
CONVECTION AND PERMEATION IN CHOLESTERIC FLUIDS
}

E. Dubois-Violette, P.-G. de Gennes

\section{To cite this version:}

E. Dubois-Violette, P.-G. de Gennes. CONVECTION AND PERMEATION IN CHOLESTERIC FLUIDS. Journal de Physique Colloques, 1975, 36 (C1), pp.C1-293-C1-298. 10.1051/jphyscol:1975149 . jpa-00216228

\section{HAL Id: jpa-00216228 https://hal.science/jpa-00216228}

Submitted on 1 Jan 1975

HAL is a multi-disciplinary open access archive for the deposit and dissemination of scientific research documents, whether they are published or not. The documents may come from teaching and research institutions in France or abroad, or from public or private research centers.
L'archive ouverte pluridisciplinaire $\mathbf{H A L}$, est destinée au dépôt et à la diffusion de documents scientifiques de niveau recherche, publiés ou non, émanant des établissements d'enseignement et de recherche français ou étrangers, des laboratoires publics ou privés. 


\title{
CONVECTION AND PERMEATION IN CHOLESTERIC FLUIDS
}

\author{
E. DUBOIS-VIOLETTE \\ Laboratoire de Physique des Solides, Université Paris-Sud, Centre d'Orsay, 91405 Orsay, France \\ and \\ P. G. DE GENNES \\ Laboratoire de Physique de la Matière Condensée, Collège de France, 75231 Paris Cedex 05, France
}

\begin{abstract}
Résumé. - On considère un échantillon planaire de cholestérique (entre deux plaques) dans lequel on impose une distribution non uniforme de température. Ceci peut être obtenu par une répartition judicieuse de sources de chaleur sur les plaques. Les vitesses $v(r)$ résultant du champ de forces de gravitation sont beaucoup plus faibles que dans les nématiques ou les liquides isotropes usuels. Cet effet est dû au processus de perméation décrit par Helfrich [1]. Une preuve expérimentale directe de la perméation pourrait être donnée par les mesures des vitesses $v(r)$.
\end{abstract}

Abstract. - We consider a planar cholesteric texture in a non uniform temperature distribution $T(\mathbf{r})$ induced by suitable heat sources on both plates. (The cholesteric pitch is assumed nearly independent of $T$.) The buoyancy forces give rise to a velocity field $\mathbf{v}(\mathbf{r})$ where $v$ is much smaller than in nematics or isotropic fluids : the slowing down is due to the permeation effect of Helfrich [1]. Measurements on $v(r)$ should lead to a direct experimental proof of the permeation process.

1. Introduction. - A number of experimental studies on the behavior of cholesterics under flow have been carried out mainly by the group at Xerox [2]. However, the conditions realised in these experiments involve strong distortion of the cholesteric planes by the flow, and their analysis is difficult. Helfrich [1] has considered theoretically a simple, and most interesting situation, where the planes are assumed fixed by suitable anchoring on the walls, as shown on figure 1. If a weak pressure gradient $\partial p / \partial z$ is applied along the normal to the planes, he showed that we should expect a flow velocity

$$
v_{z}=-\lambda_{\mathrm{p}} \frac{\partial p}{\partial z}
$$

where the permeation coefficient $\lambda_{\mathrm{p}}$ is given explicitly by

$$
\lambda_{p}=\frac{1}{\gamma_{1} q_{0}^{2}}=-\frac{1}{\pi^{2}} \frac{d^{2}}{\gamma_{1}}
$$

where $d=\pi / q_{0}$ is the half-pitch, and $\gamma_{1}$ a Leslie coefficient coupling orientation and flow [3]. Eq. (1.1) probably explains the high apparent viscosity of cholesterics, although some of the materials which were quoted at the time have later turned out to be actually smectics. More recent attempts to achieve well defined permeation flows inside a capillary with anchored planes have encountered great difficulties $\left({ }^{1}\right)$ : in practice, the

(1) Gasparoux H., private communication and CANDaU, S., private communication.

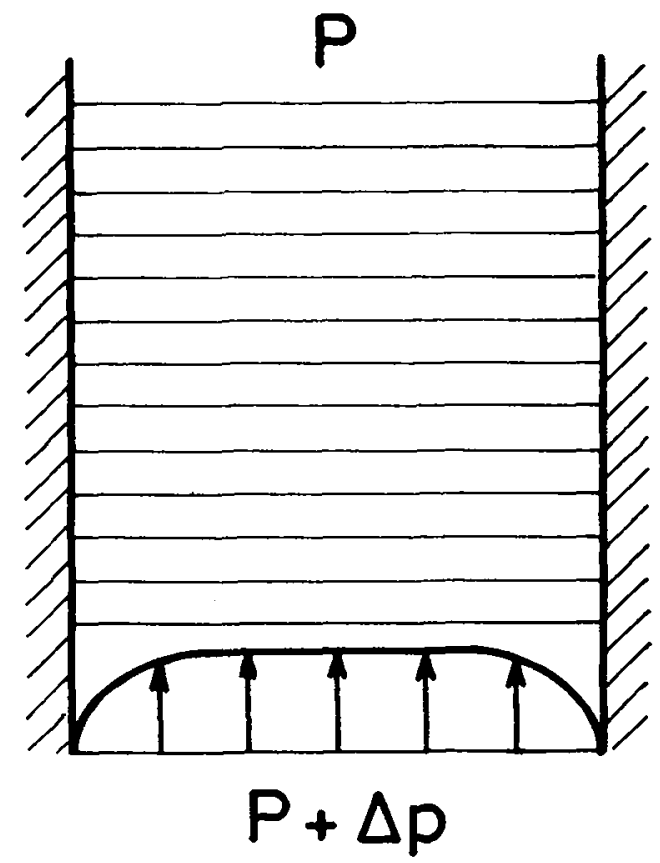

Fig. 1. - Permeation effect : one applies a vertical pressure gradient $(\nabla p)$ on a cholesteric sample with anchored planes. One expects a flow velocity $V \sim \lambda_{\mathrm{p}} \nabla p$.

planes do not remain normal to the capillary axis as soon as a significant flow is imposed. Thus, to study the permeation process, it is probably necessary to use different geometries, where the sample is flat as shown 
on figure 2 or 3 , and where the cholesteric planes are more effectively anchored.

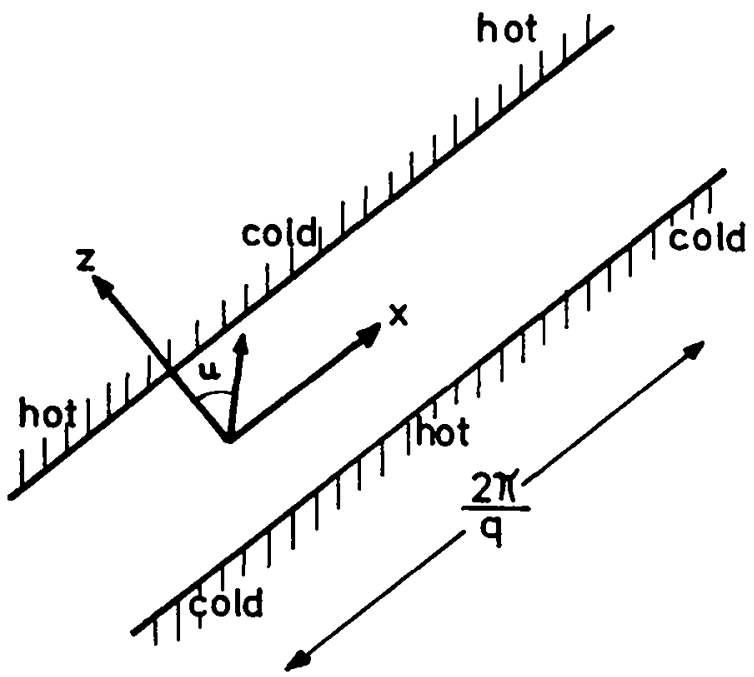

Fig. 2. - A non uniform temperature distribution is applied on a cholesteric sample. The cholesteric layers are parallel to the plates. $u$ is the angle between the vertical direction and the normal to the plates.

However, it is not easy to inject fluid inside such a cell in a well controlled fashion. This has led us to a different approach, where the cell is closed and certain loop currents are induced inside the fluid by non homogeneous external forces. The two main candidates for the external agent are an electric force (acting on injected charges) or a buoyancy force. The process of charge injection is too complex to be practical at present. We are thus left with thermal effects; we shall assume that both plates in the cell of figure 2 have a prescribed, non uniform, temperature distribution $T$, and investigate the resulting flow velocities : they could be measured in practice by Doppler techniques [4]. Section 2 defines the model. Section 3 gives the mechanical equations. Section 4 analyses the boundary layer effects. Section 5 discusses the main results.

2. The linear model. - At first sight we might be worried by the effects of the temperature $T$ on $d$ : in many cholesterol esters, $d$ will shrink if $T$ is raised even slightly. Fortunately this effect becomes very small if we use dilute solutions of a chiral agent inside a nematic matrix. These dilute systems give rather large values of $d$ (e. g. $20 \mu$ ) which will turn out to be convenient for the experiments which we have in mind.

Another possible complication could come from thermal convection : a number of remarkable convective effects have, indeed, been found in liquid crystals [5]. However, for the very slow $(10 \mu / \mathrm{s})$ motions which we have in mind, it will usually turn out that conduction still dominates on convection. Thus, we can assume that the temperature distribution $T(r)$ satisfies the steady state equation :

$$
K_{\|} \frac{\partial^{2} T}{\partial z^{2}}+K_{\perp}\left(\frac{\partial^{2} T}{\partial x^{2}}+\frac{\partial^{2} T}{\partial y^{2}}\right)=0
$$

where $K_{\|}$is the thermal conductivity along the helical axis $(z)$ and $K_{\perp}$ is the conductivity in the plane of the cholesteric layers.

We are then left with a problem of laminar flow, where the velocity field $\mathbf{v}(r)$ is a linear functional of the temperature field $T(r)\left({ }^{2}\right)$. In this linearised approximation, it is correct to assume that the layers are essentially undisturbed by the flow. Related problems of flow in a stratified medium (smectic $\mathrm{A}$ ) have been considered recently by one of us [6] using the Martin Parodi Pershan equations [7]. Similar equations hold for cholesterics [8], when they can be treated as a supercontinuum, i. e. when all characteristic lengths of the flow are large in comparison with $d$. We shall always work in this limit.

Since the problem is now linear, it will be sufficient to consider one Fourier component, i. e. a temperature distribution of the form

$$
T(x y z)=T_{q}(z) \cos q x
$$

where $q$ is an arbitrary wave vector. We shall be most interested in the limit where $q L<1$ where $L$ is the sample thickness. Eq. (2.1) allows us to write down the $z$ dependence explicitly

$$
T_{4}(z)=A \operatorname{ch} \tilde{q} z+B \operatorname{sh} \tilde{q} z
$$

where we put the origin of the $z$ coordinates at the mid plane. Here $\tilde{q}$ is defined by

$$
\tilde{q}=q\left(\frac{K_{1}}{K_{\|}}\right)^{1 / 2}
$$

and is usually comparable to $q$. We shall consider separately the even mode $(A \neq 0, B=0)$ and the odd mode $(A=0, B \neq 0)$, the latter being shown on figure 2. By suitable combination of such modes, we can adjust eq. (2.2) to any (piecewise continuous) distribution of temperatures on both plates. But in practice, if desired, it shall not be too hard to realise a simple sinewave by heating the plates with an array of evaporated metallic films. Of major interest for the experimentalist is the temperature difference between the warmest and the coldest point in the sample, which we shall call $\Delta T$ (see Fig. 5)

$$
\Delta T=\left\{\begin{array}{ll}
2 A \operatorname{ch} \tilde{q} \frac{L}{2} & \text { (even) } \\
2 B \operatorname{sh} \tilde{q} \frac{L}{2} & \text { (odd) }
\end{array}\right\} .
$$

3. Mechanical equations. - Having now specified the temperature distribution, we can write down the force balance for the fluid. In a linearised regime, it reads $\left({ }^{3}\right)$

(2) We always define $T(r)$ so that its space average vanishes.

(3) Summation over repeated indices is assumed. 


$$
\begin{aligned}
& 0=\rho \frac{\partial v_{z}}{\partial t}=-\frac{\partial p}{\partial z}+f_{z}-\frac{v_{z}}{\lambda_{p}}+\partial_{\alpha} \sigma_{\alpha z} \\
& 0=\rho \frac{\partial v_{x}}{\partial t}=-\frac{\partial p}{\partial x}+f_{x}+\partial_{\alpha} \sigma_{\alpha x} .
\end{aligned}
$$

Here $p$ is the scalar pressure, $f$ is the buoyancy force

$$
\begin{aligned}
& f_{z}=\rho g \alpha T \cos u=f \cos u \\
& f_{x}=\rho g \alpha T \sin u=f \sin u
\end{aligned}
$$

$u$ is the angle between the vertical and the helical axis $z$ (see Fig. 2), $g$ the gravitational acceleration, $\alpha$ the dilatation coefficient, and $\rho$ the density. The third term in eq. (3.1) describes the resistance to permeation, and $\lambda_{\mathrm{p}}$ is given by eq. (1.2), $\sigma_{\alpha \beta}$ is the viscous stress tensor.

As shown in [6], this term is always negligible for the $z$ component of the force equation, provided that we are in the continuum limit. On the other hand, the viscous friction cannot be omitted for motions in the layer plane, and gives in eq. (3.2) a contribution [6] :

$$
\hat{o}_{\alpha} \sigma_{\alpha x}=v_{2}\left(\frac{\partial^{2}}{\partial x^{2}}+\frac{\partial^{2}}{\partial y^{2}}\right) v_{x}+v_{3} \frac{\partial^{2} v_{x}}{\partial z^{2}}
$$

where $v_{2}$ and $v_{3}$ are two viscosities, characteristic of the cholesteric in the supercontinuum limit.

We shall naturally work in the Boussinesq approximation, and set

$$
\operatorname{div} \mathbf{v}=0 .
$$

For the two dimensional problem at hand (where all observables depend only on $x$ and $z$ ) it is convenient to enforce (3.5) by the introduction of a stream function $\psi(x, z)$

$$
\begin{aligned}
& v_{x}=\frac{\partial \psi}{\partial z} \\
& v_{z}=-\frac{\partial \psi}{\partial x} .
\end{aligned}
$$

Eliminating the pressure between eq. (3.1) (3.2) we then arrive at

$$
\begin{aligned}
\frac{1}{\lambda_{p}} \frac{\partial^{2} \psi}{\partial x^{2}}-( & \left.v_{2} \frac{\partial^{2}}{\partial x^{2}}+v_{3} \frac{\partial^{2}}{\partial z^{2}}\right) \frac{\partial^{2} \psi}{\partial z^{2}}= \\
& =\frac{\partial f_{x}}{\partial z}-\frac{\partial f_{z}}{\partial x}=\sin u \frac{\partial f}{\partial z}-\cos u \frac{\partial f}{\partial x} .
\end{aligned}
$$

From now on we shall restrict our attention to the case where $q L<1$. Then, the variations along $x$ are slow in comparison with the variations along $z$, and eq. (3.7) may be further simplified to

$$
\frac{\partial^{2} \psi}{\partial x^{2}}-\frac{1}{K^{2}} \frac{\partial^{4} \psi}{\partial z^{4}}=\lambda_{\mathrm{p}}\left(\sin u \frac{\partial f}{\partial z}-\cos u \frac{\partial f}{\partial x}\right) .
$$

Here, following [6], we have introduced a characteristic length $K^{-1}$ through

$$
K^{2}=\frac{1}{v_{3} \lambda_{p}}=\frac{\gamma_{1}}{v_{3}} q_{0}^{2}
$$

In practice, $K$ will be comparable to $q_{0}$ (or $K^{-1}$ comparable to $d$ ). Eq. (3.8), together with the boundary conditions

$$
\begin{aligned}
\psi & =0 \\
\frac{\partial \psi}{\partial z} & =0
\end{aligned}
$$

on both plates, defines entirely the flow field.

4. The boundary layer approximation. - The operator occurring on the left hand side of eq. (3.8) has been discussed in [6]. Here we shall summarize the discussion in simplified terms. For the case at hand, we have

$$
\frac{\partial^{2} \psi}{\partial x^{2}}=-q^{2} \psi
$$

Inspection of (3.8) suggests the introduction of a characteristic length such that

$$
\delta^{2}=1 / K q .
$$

Two regimes are of interest, depending on the ratio $\delta / L$.

If $\delta>L$, the term $\partial^{2} \psi / \partial x^{2}$ becomes negligible in (3.8) and we are left with

$$
\frac{\partial^{4} \psi}{\partial z^{4}}=\frac{1}{v_{3}}\left(\cos u \frac{\partial f}{\partial x}-\sin u \frac{\partial f}{\partial z}\right) .
$$

The permeation coefficient $\lambda_{p}$ drops out of eq. (4.2) : when $\delta>L$, we have a simple laminar flow inside the layers, and the behavior resembles an isotropic fluid. Clearly, this is not the situation of interest for our purposes. Thus, from now on, we shall restrict our attention to the opposite limit

$$
\delta<L \text { or } K q L^{2}>1 .
$$

Then, in most of the sample volume, we can neglect the term $\partial^{4} \psi / \partial z^{4}$ of eq. (3.8) and write

$$
-q^{2} \psi=\lambda_{\mathrm{p}}\left(\sin u \frac{\partial f}{\partial z}-\cos u \frac{\partial f}{\partial x}\right) .
$$

From this we can construct the velocities

$$
v_{x}=\frac{\partial \psi}{\partial z}=\frac{\lambda_{\mathrm{p}}}{q^{2}}\left(\cos u \frac{\partial^{2} f}{\partial x \partial z}-\sin u \frac{\partial^{2} f}{\partial z^{2}}\right) .
$$

We can transform this slightly because $f$ is proportional to $T$ and thus satisfies eq. (2.1)

$$
\frac{\partial^{2} f}{\partial z^{2}}=-\frac{K_{\perp}}{K_{\|}} \frac{\partial^{2} f}{\partial x^{2}}=\frac{K_{1}}{K_{\|}} q^{2} f
$$

giving

$$
\frac{v_{x}}{\lambda_{p}}=+\cos u \frac{1}{q^{2}} \frac{\partial^{2} f}{\partial x \partial z}-\sin u \frac{K_{1}}{K_{\|}} f .
$$

Similarly, for the component normal to the layers, we find

$$
\frac{v_{z}}{\lambda_{\mathrm{p}}}=f \cos u+\frac{1}{q^{2}} \frac{\partial^{2} f}{\partial x \partial z} \sin u .
$$


Eq. (4.6), (4.7) give the solution nearly everywhere. However, this solution cannot be correct near the walls, where $\mathbf{v}=0$. The adjustment may be shown to take place in a boundary layer of thickness $\delta$ [6].

5. Inner structure of the boundary layer. 5.1 VeRTICAL LAYERS. - Let us first restrict our attention to the vertical configuration $(u=\pi / 2)$ and to the odd temperature mode $T=B \operatorname{sh} \tilde{q z}$ which will turn out to be the more favourable for our purpose. The shear function which satisfies eq. (3.8) and the boundary conditions (3.10) is

$\psi=\left(\psi_{0}+a \operatorname{ch} k z \cos k z-b \operatorname{sh} k z \sin k z\right) \cos q x$ where $\psi_{0}=-\frac{\rho g \alpha \lambda}{q} \frac{\partial T}{\partial z}$ is a particular solution of eq. (3.8) in the limit defined by (4.3).

Here

$$
\begin{aligned}
a=\frac{\rho g \alpha \hat{\lambda}_{\mathrm{p}}}{q} & B\left(\frac{K_{\perp}}{K_{\mathrm{ll}}}\right)^{1 / 2} 2 \frac{\operatorname{ch}\left(\frac{\tilde{q} L}{2}\right)}{\mathrm{e}^{\mu}} \times \\
& \times\left(\cos \theta+\sin \theta-\frac{q}{k}\left(\frac{K_{\perp}}{K_{\|}}\right)^{1 / 2} \frac{\operatorname{sh}\left(\frac{\tilde{q} L}{2}\right)}{\operatorname{ch}\left(\frac{\tilde{q} L}{2}\right)} \sin \theta\right)
\end{aligned}
$$$$
b=\frac{\rho g \alpha \lambda_{\mathrm{p}}}{q} B\left(\frac{K_{\perp}}{K_{\|}}\right)^{1 / 2} 2 \frac{\operatorname{ch}\left(\frac{\tilde{q} L}{2}\right)}{\mathrm{e}^{u}} \times
$$$$
\times\left(\cos \theta-\sin \theta-\frac{q}{K}\left(\frac{K_{\perp}}{K_{\|}}\right)^{1 / 2} \frac{\operatorname{sh}\left(\frac{\tilde{q} L}{2}\right)}{\operatorname{ch}\left(\frac{\tilde{q} L}{2}\right)} \cos \theta\right)
$$

$$
k=\frac{\sqrt{2}}{2}(q K)^{1 / 2} \text { and } \theta=\frac{k L}{2} \gg 1 .
$$

Except in the boundary layer of thickness $\delta=k^{-1}$ the flow follows from the stream function $\psi_{0}$ :

$$
\begin{aligned}
& v_{x}=\frac{\partial \psi_{0}}{\partial z}=-\rho g \alpha \lambda_{\mathrm{p}} B\left(\frac{K_{\perp}}{K_{\|}}\right) \operatorname{sh} \tilde{q} z \cos q x \\
& v_{z}=-\frac{\partial \psi_{0}}{\partial x}=-\rho g \alpha \hat{\lambda}_{\mathrm{p}} B\left(\frac{K_{\perp}}{K_{\|}}\right)^{1 / 2} \operatorname{ch} \tilde{q} z \sin q x .
\end{aligned}
$$

In order to get the velocity field in the boundary layer, one expands expressions $(5.1),(5.2),(5.3)$ in powers of

$$
\varepsilon=\frac{k L}{2}-k z \text {. }
$$

After some rearranging, one obtains :

$$
\begin{aligned}
& v_{x}=\rho g \alpha \lambda_{\mathrm{p}} B\left(\frac{K_{\perp}}{K_{\|}}\right)^{1 / 2} \operatorname{ch}\left(\frac{\tilde{q} L}{2}\right) \frac{2 k}{q} \varepsilon \cos q x . \\
& v_{z}=-\rho g \alpha \lambda_{\mathrm{p}} B\left(\frac{K_{\perp}}{K_{\|}}\right)^{1 / 2} \operatorname{ch}\left(\frac{\tilde{q} L}{2}\right) \varepsilon^{2} \sin q x .
\end{aligned}
$$

Let us remark that the second terms in parentheses of eq. (5.2), (5.3) must be included (even though of order $\left.q^{2} L / k<1\right)$ in order to cancel $\psi_{0}$ and $\partial \psi_{0} / \partial z$ at the boundaries. The flow pattern described by eq. (5.1)(5.6) is shown in figure 3.

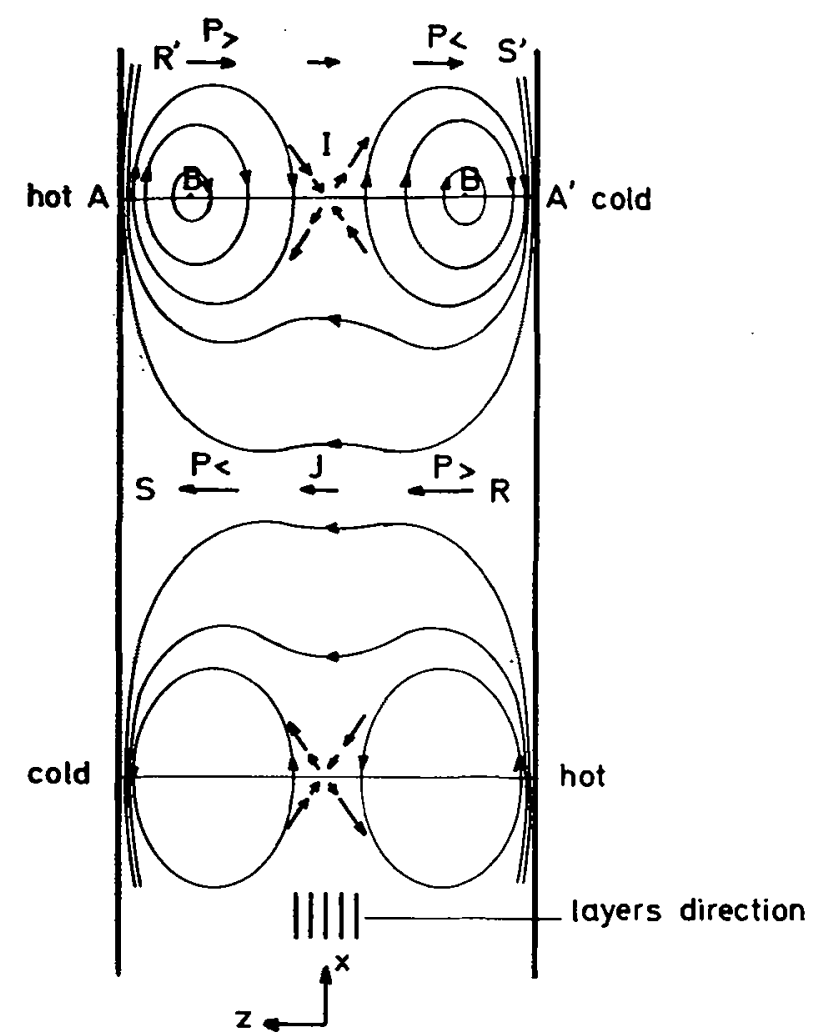

FIG. 3. - Qualitative flow pattern in a vertical geometry. Along an horizontal path, $\mathbf{A A}^{\prime}$, one meets : a boundary layer between AB (and $A^{\prime} B$ ) with high velocities directed parallel to the buoyancy forces (see Fig. 4); an inner region where viscous forces along the vertical are negligible : the buoyancy force is balanced by the pressure gradient and the velocities are opposite in sign to the buoyancy force.

The pattern is extremely different from what we would have in an isotropic liquid. Starting from a hot region on one plate, and moving inwards (on the ABI path of Fig. 3) we meet :

a) Between A and B a boundary layer of width $\delta$ with high velocities directed upwards parallel to the buoyancy forces.

b) Between $B$ and the midpoint $I$ an inner region where the velocity in the layer plane is opposite in sign to the buoyancy force.

This unusual behaviour may be understood as follows : in the inner region, the vertical pressure gradient exactly balances the buoyancy force (viscous forces along the vertical being negligible). Thus the pressure is large at points $R, R^{\prime}$ and low at $S$ and $S^{\prime}$. The pressure gradient between $R$ and $S$ induces a permeation flow from $R$ towards $S$. The corresponding permeation velocity is nearly uniform between $R$ and $S$, but slightly larger at $R$ or $S$ than at the midpoint $J$ 
(Fig. 4). The resulting current lines must then close up in the inner region, and thus forces a downward flow in the region between $B$ and $I$.
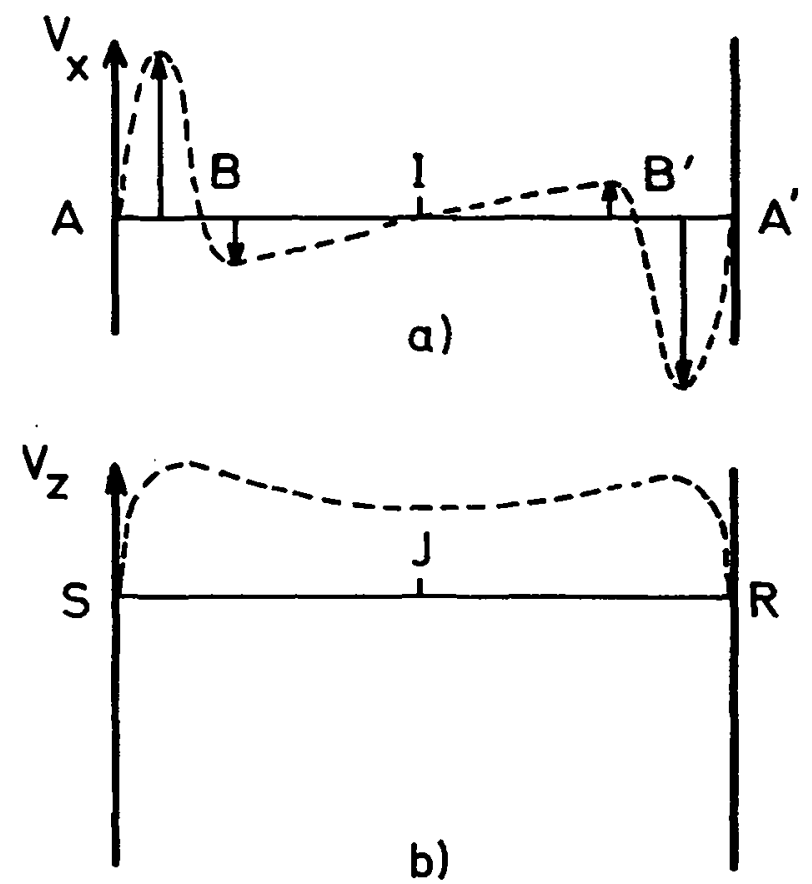

Frg. 4. - a) Profile of a vertical velocity $\left(V_{x}\right)$ on a line $\mathrm{AA}^{\prime}$; b) Profile of the horizontal velocity $\left(V_{z}\right)$ on a line SR.

5.2 HORIzONTAL LAYERS. - Results for the geometry $(u=0)$ are easily deduced from the preceding configuration and lead to :

$$
\begin{aligned}
& a=\frac{\rho g \alpha \lambda_{\mathrm{p}}}{q} B 2 \frac{\operatorname{sh}\left(\frac{\tilde{q} L}{2}\right)}{\mathrm{e}^{\mu}} \times \\
& \quad \times\left(\cos \theta+\sin \theta-\frac{q}{k}\left(\frac{K_{\perp}}{K_{\|}}\right)^{1 / 2} \frac{\operatorname{ch}\left(\frac{\tilde{q} L}{2}\right)}{\operatorname{sh}\left(\frac{\tilde{q} L}{2}\right)} \sin \theta\right) \\
& b=\frac{\rho g \alpha \lambda_{p}}{q} B 2 \frac{\mathrm{e}^{\mu}}{\operatorname{sh}\left(\frac{\tilde{q} L}{2}\right)} \times \\
& \quad \times\left(\cos \theta-\sin \theta-\frac{q}{k}\left(\frac{K_{\perp}}{K_{\|}}\right)^{1 / 2} \frac{\operatorname{ch}\left(\frac{\tilde{q} L}{2}\right)}{\operatorname{sh}\left(\frac{\tilde{q} L}{2}\right)} \cos \theta\right) .
\end{aligned}
$$

The velocities in the bulk are :

$$
\begin{aligned}
& v_{x}=\frac{\partial \psi_{0}}{\partial z}=-\rho g \alpha \lambda_{\mathrm{p}} B\left(\frac{K_{\perp}}{K_{n}}\right)^{1 / 2} \operatorname{ch} \tilde{q} z \sin q x \\
& v_{z}=\frac{\partial \psi_{0}}{\partial x}=\rho g \alpha \lambda_{\mathrm{p}} B \operatorname{sh} \tilde{q} z \cos q x
\end{aligned}
$$

and in the boundary layer

$$
\begin{aligned}
& v_{x}=\rho g \alpha \lambda_{\mathrm{p}} B 2 \frac{k}{q} \operatorname{sh} \frac{\tilde{q} L}{2} \varepsilon \sin q x \\
& v_{z}=\rho g \alpha \lambda_{\mathrm{p}} B \operatorname{sh} \frac{\tilde{q} L}{2} \varepsilon^{2} \cos q x .
\end{aligned}
$$

The flow pattern is shown on figure 5 .

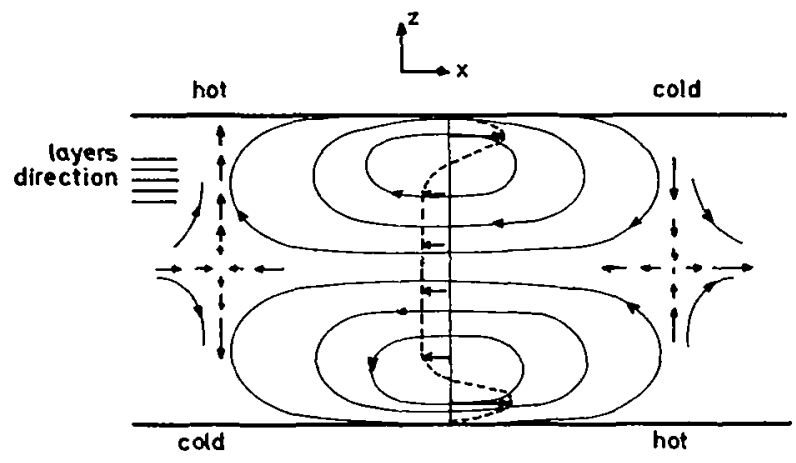

FIG. 5. - Qualitative flow pattern in a horizontal geometry.

6. Orders of magnitude. - 6.1 INNER REGION. For a general value of $u$, we see that such component as $v_{x}, v_{z}$ is a superposition of two terms :

a) a term of order $\lambda_{\mathrm{p}} f$, i. e. comparable to what we would have in the case of figure 1 , with the buoyancy force replacing the pressure gradient;

b) a term of order $\lambda_{\mathrm{p}} \frac{1}{q^{2}} \frac{\partial^{2} f}{\partial x \partial z}$.

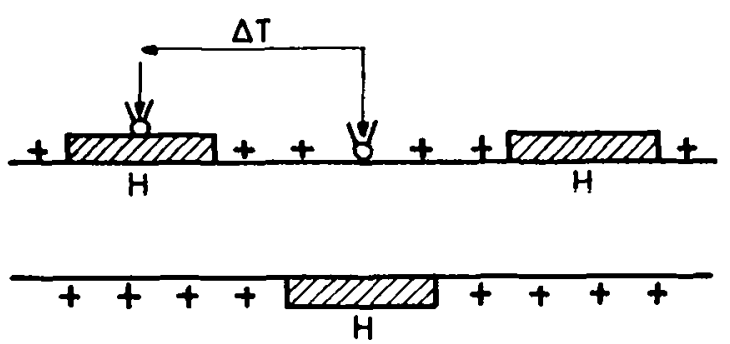

Fig. 6. - Practical relization of the heat distribution.

To compare these two terms, we return to eq. (2.3) giving the temperature distribution. For the even mode, from eq. (3.3) and (2.5) we find

$$
f=\rho g \alpha \frac{\Delta T}{2 \operatorname{ch}(\tilde{q} L / 2)} \operatorname{ch}(\tilde{q} z) \cos (q x)
$$

$$
\begin{aligned}
\frac{1}{q} \frac{\partial^{2} f}{\partial x \partial z} & =-\rho g \alpha \frac{\Delta T}{2 \operatorname{ch}(\tilde{q} L / 2)}\left(\frac{K_{1}}{K_{\|}}\right)^{1 / 2} \operatorname{sh}(\tilde{q} z) \sin (q x) \\
& \cong-\frac{\rho g \alpha \Delta T}{2} \frac{K_{\perp}}{K_{\|}} q z \sin (q x) \quad(q L \ll 1) .
\end{aligned}
$$


Omitting the factors $\cos (q x)$, sin $(q x)$, and taking the ratio of the amplitudes, we have

$$
\left|\frac{1}{q^{2} f} \frac{\partial^{2} f}{\partial x \partial z}\right| \sim \frac{K_{\perp}}{K_{\|}} q z \sim q L<1
$$

(even mode).

Thus, for the even mode, it is the term (a) which dominates. For the odd mode :

$$
\begin{aligned}
& f=\rho g \alpha \frac{\Delta T}{2 \operatorname{sh}(\tilde{q} L / 2)} \operatorname{sh}(\tilde{q} z) \cos q x \cong \\
& \cong \rho g \alpha \Delta T \frac{z}{L} \cos q x \quad(\tilde{q} L \ll 1) \\
& \left|\frac{1}{q^{2} f}-\frac{\partial^{2} f}{\partial x \partial z}\right| \sim \frac{1}{q z} \sim \frac{1}{q L}>1 \quad \text { (odd mode) }
\end{aligned}
$$

Thus, in the limit $q L<1$, the odd mode is more favourable if we wish to produce sizeable velocities without imposing prohibitely large intervals $\Delta T$. The resulting two main situations (corresponding to $u=\pi / 2$ and $u=0$ ) are shown on figures 3 and 5 . Note that, in both cases, the largest velocity component in the inner region is horizontal (normal to $f$ ). The order of magnitude of this large component is

$$
\left.\begin{array}{rl}
V_{\mathrm{M}} & =\lambda_{\mathrm{p}} \rho g \alpha \Delta T \cdot \frac{1}{q_{\mathrm{L}}} \\
& =\frac{\rho g \alpha \Delta r}{\gamma_{1} q_{0}^{2} q L}
\end{array}\right\}\left(\begin{array}{l}
\text { odd mode } \\
\text { inner region }
\end{array}\right) .
$$

Eq. (6.4) shows that the velocity $v$ is measurable only if the cholesteric wave vector $q_{0}=\pi / d$ is small ( $d$ large). This in turn forces one to have a large sample thickness $L$ if we want to satisfy (4.3) while keeping $q L<1$. A typical choice of numbers is

$$
\begin{aligned}
& L=300 \mu \\
& d=10 \mu \quad\left(q_{0}=3 \times 10^{3} \mathrm{~cm}^{-1}\right)
\end{aligned}
$$

$q L=1 / 3$ corresponding to a wavelength $2 \pi / q=6 \mathrm{~mm}$

$$
\begin{aligned}
K & \sim q_{0} \sim 3 \times 10^{3} \mathrm{~cm}^{-1} \\
\gamma_{1} & \sim 10^{-1} \text { poise }
\end{aligned}
$$

$$
\begin{aligned}
\alpha & =1 / 300 \\
\Delta T & =3^{\circ} \\
\rho & =1 \mathrm{~g} / \mathrm{cm}^{3} .
\end{aligned}
$$

This gives $\delta^{2} / L^{2} \sim 0.07, v_{M} \sim 0.3 \mu / \mathrm{s}$.

6.2 BOUNDARY LAYER. - As shown by the numerical values above, the velocities in the inner region are somewhat too small to be measured conveniently (even with modern optical techniques). On the other hand, the velocities in the boundary layer are significantly larger. Also, in most practical instances, the thickness $\delta$ of the boundary layer is sizeable, and optical measurements in this region are conceivable. In this region, the order of magnitude of the velocity is

$$
\widetilde{V}_{\mathrm{M}} \cong \kappa \delta V_{\mathrm{M}}
$$

Keeping the same numerical values, we have $\kappa \delta \sim 30$ and $\widetilde{V}_{M} \sim 9 \mu / \mathrm{s}$.

With such values of $\widetilde{V}_{M}$, the experiment appears feasible (although difficult) since there exists now optical techniques using small solid particles as a label [4] $\left(^{4}\right.$ ). Larger samples (say $L \sim 1 \mathrm{~mm}$ ) would allow for larger velocities but they may be difficult to prepare in single domains. The required temperature distribution may be approximately realised by a set of heating films as shown on figure 5. In fact, the most interesting procedure would be to apply a magnetic field and to unwind the helix : qualitatively this may be described as a decrease of $q_{0}$, leading to an increase of $v_{M}$; finally we may mention that, when a nematic phase is reached, formula $(6.4)$ is still qualitatively correct (in the limit of small $\Delta T$ ) if we replace $q_{0}$ by const. $1 / L$ : in the example above, this would represent an increase by a factor 100 . However, this limit is not too meaningful since, for a nematic with our values of $\Delta T, \tilde{v}_{M}$ becomes of order $700 \mu / \mathrm{s}$ and convection becomes important.

Acknowledgments. - We have greatly benefited from conversations with various members of the Orsay group, and particularly with $L$. Léger, $O$. Parodi and A. Rapini.

(4) Bergé, P., Dubois, M., private communication.

\section{References}

[1] Helfrich, W., Phys. Rev. Lett. 23 (1969) 372.

[2] Pochan, J. M., Erhardt, P. F., Phys. Rev. Lett. 27 (1971) 790 ;

Marsh, D., Pochan, J. M., J. Chem. Phys. 58 (1973) 2835.

[3] Leslie, F., Arch. Rat. Mech. Anal. 28 (1968) 265.

[4] YeH, Y, Cummins, H. Z., Appl. Phys. Lett. 4 (1964) 176 ; Foreman, J. W., George, E. W., Jetron, J. L., Lewis, R. D. Thorton, J. P., Watson, H. J., J. of Quant. Electr. 2 (1966) 260 ;
Jackson, D. A., Paul, D. M., Phys. Lett. 32A (1970) 77.

[5] Dubors-Violette, E., Guyon, E., Pieranski, P., Mol. Cryst. \& Liqu. Cryst., Liqu. Cryst. Conf. Kent (1972);

Dubois-Violette, E., Solid State Commun. 14 (1974) 767.

[6] De Gennes, P. G., to be published.

[7] Martin, P. C., Pershan, P. S., Parodi, O., Phys. Rev. 6A (1972) 2401.

[8] Lubensky, T., Phys. Rev. 6A (1972) 452 ; Phys. Rev. Lett. 29 (1972) 206 